\title{
Perspective \\ Detection Methods Fit-for-Purpose in Enforcement Control of Genetically Modified Plants Produced with Novel Genomic Techniques (NGTs)
}

\author{
Alexandra Ribarits ${ }^{1, *}$, Frank Narendja ${ }^{2}$, Walter Stepanek ${ }^{1}$ and Rupert Hochegger ${ }^{1}(\mathbb{D}$ \\ 1 Austrian Agency for Health and Food Safety, Spargelfeldstraße 191, 1220 Vienna, Austria; \\ walter.stepanek@ages.at (W.S.); rupert.hochegger@ages.at (R.H.) \\ 2 Environment Agency Austria, Spittelauer Lände 5, 1090 Vienna, Austria; \\ frank.narendja@umweltbundesamt.at \\ * Correspondence: alexandra.ribarits@ages.at
}

check for updates

Citation: Ribarits, A.; Narendja, F.; Stepanek, W.; Hochegger, R.

Detection Methods Fit-for-Purpose in Enforcement Control of Genetically

Modified Plants Produced with Novel Genomic Techniques (NGTs). Agronomy 2021, 11, 61.

https://doi.org/10.3390/

agronomy11010061

Received: 30 November 2020 Accepted: 27 December 2020 Published: 30 December 2020

Publisher's Note: MDPI stays neutral with regard to jurisdictional clai$\mathrm{ms}$ in published maps and institutional affiliations.

Copyright: (C) 2020 by the authors. Licensee MDPI, Basel, Switzerland. This article is an open access article distributed under the terms and conditions of the Creative Commons Attribution (CC BY) license (https:// creativecommons.org/licenses/by/ $4.0 /)$

\begin{abstract}
The comprehensive EU regulatory framework regarding GMOs aims at preventing damage to human and animal health and the environment, and foresees labelling and traceability. Genomeedited plants and products fall under these EU GMO regulations, which have to be implemented in enforcement control activities. GMO detection methods currently used by enforcement laboratories are based on real-time PCR, where specificity and sensitivity are important performance parameters. Genome editing allows the targeted modification of nucleotide sequences in organisms, including plants, and often produces single nucleotide variants (SNVs), which are the most challenging class of genome edits to detect. The test method must therefore meet advanced requirements regarding specificity, which can be increased by modifying a PCR method. Digital PCR systems achieve a very high sensitivity and have advantages in quantitative measurement. Sequencing methods may also be used to detect DNA modifications caused by genome editing. Whereas most PCR methods can be carried out in an enforcement laboratory with existing technical equipment and staff, the processing of the sequencing data requires additional resources and the appropriate bioinformatic expertise.
\end{abstract}

Keywords: GMO; genome editing; detection methods; regulation; enforcement

\section{Introduction}

In the EU, when placing on the market genetically modified (GM) crops and derived food and feed, a rigorous regulatory framework that is based on the precautionary principle aims at protecting consumers and the environment [1]. Regardless of safety aspects, the possibility to choose between products with and without genetic modification techniques in the production process is important to consumers, as well as farmers and food manufacturers. GMO-free production is also essential for products that are declared as GM-free or produced under organic farming concepts. EU policy on GMOs is comprehensive. It deals in detail with the whole agro-food chain, encompassing all stages of production, from the development of GMOs, their release and cultivation, marketing, labelling, and monitoring up to the point where these products are consumed by humans and animals [2-5]. Enforcement encompasses protection of the market from the presence of unauthorized GMOs and general requirements on labelling. Labelling and traceability requirements are specified in regulations (EC) 1829/2003, (EC) 1830/2003, and (EU) 619/2011 [3,4,6]. A labelling threshold of $0.9 \%$ for the adventitious or technically unavoidable presence of GMOs in products is set in the European legislation framework (labelling threshold, Regulation (EC) 1829/2003, implementation of Regulation (EC) 1830/2003 [3,4]). Additionally, a quantitative determination of GMOs in amounts as low as $0.1 \%$ should be possible for the adventitious presence of a GMO, for which an authorization procedure is pending or the authorization of which has expired [6]. For unauthorized GMOs without a pending 
application, zero tolerance applies (Regulation (EU) 619/2011). The reliability of test results is crucial in enforcement control, as they are usually associated with corresponding measures. According to Regulation (EC) 625/2017, uniform control measures are required in the EU member states [7]. All EU member states have to designate one or more national reference and official control laboratories to carry out enforcement and official controls. Their competent authorities have appointed enforcement "national reference laboratories" (NRLs) and official laboratories to perform analyses for the presence of authorized and unauthorized GMOs in food, feed, and seed products [8]. These laboratories have the expertise, equipment, infrastructure, and staff to perform analyses during official controls. In the EU, enforcement laboratories apply PCR-based methods to detect DNA sequences.

On 25 July 2018, the European Court of Justice (ECJ) ruled "that organisms obtained by means of techniques/methods of mutagenesis constitute GMOs" within the meaning of Article 2(2) of Directive 2001/18/EC [9]. According to this ruling, market releases of organisms developed using new mutagenesis techniques, which have appeared or have been mostly developed since Directive 2001/18/EC was adopted, need to comply with its legal obligations. In light of the ECJ judgment, the Council of the EU has requested the Commission to submit a study regarding the status of Novel Genomic Techniques (NGTs) under Union law [10]. NGT is a collective term for techniques capable of changing the genetic material of an organism, and that have emerged or have been developed since 2001. Among these, techniques commonly known as genome editing (e.g., the widely known CRISPR/Cas system) have received the most attention globally in recent years. They allow the targeted and precise modification of the nucleotide sequence of the genome of diverse organisms, including plants. According to the current state of knowledge, NGTs and in particular genome editing represent a significant improvement in precision and efficiency compared to previous gene modification and gene transfer procedures. In particular, genome editing allows defined site specificity when altering plant genomes [11].

Generally, the detection of GMOs may be accomplished by different analytical approaches of increasing specificity: species/taxon-specific, element-specific, constructspecific, and event-specific methods [12,13]. Construct- and event-specific detection methods commonly lead to the identification of a specific GMO. The availability of suitable detection methods is the pivotal element to enable enforcement of the EU regulatory framework for GMOs. Classic GM plants are characterized by the random insertion of foreign DNA segments, and detection makes use of this characteristic. In contrast, a DNA sequence altered by genome editing can be detected, but this does not necessarily include the decision upon the technical background of a modification or the underlying process, e.g., using a genome editing technique. Furthermore, it cannot be excluded that the DNA modification occurred spontaneously or was generated by random mutagenesis. This article examines which existing and upcoming detection methods are suitable to detect typical NGT-related modifications and are potentially fit for the purpose of meeting the regulatory requirements. PCR and sequencing methods were analyzed for their potential regarding practical application in official enforcement control. The analysis is complemented by pointing out the specific needs of enforcement laboratories, and whether the selected methods could be integrated in existing testing strategies in the near future.

\section{Standards and Requirements in Enforcement Control}

The most common method applied to detect GMOs is PCR, which is routinely used for various applications, including allergen analysis or the determination of pathogens. To date, methods based on the principle of quantitative real-time PCR (qPCR) have been the gold standard for the detection of GMOs. The technical equipment to perform qPCR requires the availability of a real-time PCR device. Such devices, as well as the corresponding qualification of the personnel, are available in many official enforcement laboratories. Consequently, to date, all reference methods of the EURL-GMFF make use of this methodology [14]. Accordingly, reference methods are collected in a database (GMOMETHODS; [15]) that is hosted by the European Union Reference Laboratory for Genetically Modified Food 
and Feed (EURL-GMFF) at the Joint Research Centre (JRC) of the European Commission. Typical genetic elements found in classic GMOs are detected in the so-called matrix-based screening approach $[16,17]$, the implementation of which is supported by the JRC GMOMatrix web application [14]. Accordingly, reference methods are collected in a database (GMOMETHODS; [15]) that is hosted by the European Union Reference Laboratory for Genetically Modified Food and Feed (EURL-GMFF) at the Joint Research Centre (JRC) of the European Commission. Sequence elements commonly found in GMO constructs, however, are not expected to be present in plants modified by certain NGTs pursuing a transgene-free strategy [18]. Similar to classic GMOs lacking these elements, such plants and products cannot be tracked down by screening approaches [12].

For classic GMOs, event-specific methods may be developed based on the unique combination of inserted DNA and the recipient genome [14]. The development of suitable detection methods for plants produced using NGTs can be much more challenging, in particular with regard to genome-edited plants and products [19]. As genome editing may result in a single base pair alteration, the development of a detection method becomes even more challenging. Nonetheless, Chhalliyil et al. (2020) recently showed that it is feasible to develop a detection method for a known single base pair edit [20].

Enforcement laboratories as well as official control laboratories should be accredited for the use of suitable methods according to the standard EN ISO/IEC 17025, "General requirements for the competence of testing and calibration laboratories" [21], to allow them to produce reliable analytical data [7]. In addition, in many cases, EU legislation (e.g., Regulation (EU) 625/2017 [7]) asks for accreditation of enforcement laboratories for the respective method according to international standards.

Method validation is necessary before a method is ready to be implemented in enforcement laboratories [13]. As an essential part of the process that regulates the introduction of new GMOs as food and/or feed on the market, the validation of an analytical method follows internationally accepted protocols. Upon validation, they assure reliable, reproducible, sensitive, and accurate determination of GMOs [13]. EURL-GMFF is legally mandated to assess the validation procedure [15], and is assisted by the European Network of GMO Laboratories (ENGL; [8]). The latter has issued a guidance document on minimum performance requirements (MPR; [22]). Validation comprises an examination of method-specific characteristics, including specificity, limits for detection, and quantification [12]. The actual implementation of specific performance requirements differs depending on the purpose of the analysis. Criteria for the validation of methods to detect NGTs have not been published so far.

Reference material is needed for diverse purposes [12,23]. Sufficiently characterized reference material is required as a positive control to confirm the result of a detection reaction. In addition, the validation of quantitative methods requires the measurement to be traced back to a unit of the international system of units (e.g., to percentages by weight of a certified reference material). Finally, certified reference material with defined proportions of sequences to be detected is required for quantification. To this end, the reference material serves as a calibrant when a standard qPCR approach is used (see, e.g., [17]).

\section{Detection Methods Fit-for-Purpose}

Method-specific properties like specificity and sensitivity are important performance parameters of any method applied in an enforcement control laboratory. It is important that the detection method is specific enough to detect modifications introduced by genome editing in a highly reliable manner. Additionally, a suitable method needs to detect specifically the sequence of interest, even in complex samples of different components (e.g., food or compound feed), and should not show any interference with other genomic sequences present.

Genome editing techniques can be designed to target only a single nucleotide producing a single nucleotide variant (SNV), which is the most challenging class of genome edits to detect $[19,20]$. In order to differentiate between a modified and an unmodified sequence, 
the specificity of a detection system for the sequence of interest is of critical importance. Methods particularly suitable for detecting an SNV must meet advanced specifications regarding the performance of the assay. The aim is to increase the specificity of the detection system in order to reliably detect even minimal differences in the nucleotide sequence, such as SNVs.

Probe-based qPCR technologies like Taqman have some limitations regarding the reliable detection of SNVs. Analysis of genome-edited plants shows that, in many cases, sequence insertions or deletions (InDels) of several base pairs may result from the genome editing process. For these types of modification, this robust and well-established technology is suitable to test for specific NGT modifications.

One possibility to increase the specificity of primers and probes in a PCR is the use of base analogs that increase the stability of the base pairing. For qPCR, locked nucleic acids (LNAs) are commonly used for this purpose, where the increased stability of the base pairing is mediated by a modified structure in the ribose unit. By using these LNA analogs at the position of the NGT-modified base, the specificity of the primers or probes can be increased to such an extent that efficient amplification is only possible with the modified target sequence [24-27]. Recently, an LNA-based detection system for SNVs present in SU (sulfonylurea and imidazolinone herbicide) tolerant canola variants has been developed [20]. It allows a sensitive detection of the tested genome-edited canola.

Another promising PCR technology, which has the potential to significantly increase the specificity of a test system, is RNaseH-dependent real-time PCR [28]. A comparison of established PCR methods [29,30] for SNV differentiation with RNaseH-dependent real-time PCR shows that RNaseH-dependent real-time PCR has a much better specificity for the desired modification, and the tested SNVs can be separated more effectively.

With digital droplet PCR (ddPCR), a higher specificity can be achieved compared to the qPCR, since PCR inhibitors, as well as the competition between background DNA and target molecules, hardly play a role [31-33].

In the EU authorization and control context, it is also required that analytical methods reach a desired level of sensitivity. Real-time PCR systems generally achieve a detection limit of 5-10 copies of the target sequence. Data from the literature show that the sensitivity and detection limits of a PCR using LNA primers and RNaseH-dependent real-time PCR approaches are comparable to those of conventional PCR or qPCR [20,34]. Due to the high number of individual reactions that can be evaluated separately when using ddPCR, it is possible to achieve a very high sensitivity of the method.

Given a sufficient specificity and sensitivity of the test method, PCR methods like RNaseH-dependent real-time PCR or LNA-PCR have the potential to provide quantitative results of required quality, even for target sequences carrying an SNV. A recent publication has demonstrated that a reliable quantification of genome-edited material is feasible by applying a PCR approach using an LNA modified primer. In the previously mentioned example of the detection of SU canola, genome-edited canola could be reliably quantified down to a mass fraction of $0.1 \%$ [20].

Concerning quantification, ddPCR provides some essential advantages compared to classical qPCR methods. Its increased specificity and sensitivity combined with calibrationindependent measurement makes ddPCR a highly promising technology for the quantitative determination of genome-edited material. Due to the efficient performance of the described PCR techniques, it can be assumed that genome-edited material can be detected even in an excess of other sequences, as is the case in mixed samples (e.g., food or feed).

Sequencing methods are also suitable for detecting DNA modifications, like SNVs and InDels, that typically occur in genome-edited plants. Furthermore, the detection of SNVs and InDels is possible in composite sample material with a sensitivity of less than $1 \%$, but requires a minimum sequencing depth (30 to 50 times of coverage). Homologous sequence regions and repetitive sequences of the products under investigation can impair the quality of the sequencing results [35]. 


\section{Discussion}

The EURL-GMFF has a large number of standardized reference PCR methods for the detection of transgenic constructs and of classical GMOs available [15], which in principle might be adapted to detect genome-edited plants. A key criterion of selecting methods potentially suitable for tracking genome-edited products is that they have been shown to successfully detect SNVs [20]. To be fit for purpose, a method used in an enforcement laboratory should meet the minimum performance criteria and correlate with the environmental conditions prevailing in a laboratory. This includes the technical equipment and the expertise of staff to implement new test strategies if necessary.

Whether a method can be classified as fit for purpose does not only depend on methodspecific properties like specificity and sensitivity. It is equally important to evaluate the specific criteria and requirements of enforcement control, regulatory needs, consumer expectations, and cost efficiency. In order to ensure fast, smooth, and cost-efficient implementation in enforcement control, methods for the detection of NGT products should be based on existing analytical systems wherever possible. When evaluating fit-for-purpose detection methods, it is thus advisable to take into account the requirements regarding the expertise of the employees and the necessary equipment of the laboratory considering the current testing approach. Ideally, appropriate methods can be established in a laboratory without large investments in equipment or personnel resources.

The analysis of different detection methods shows that some PCR methods are suitable for detecting sequence differences, even if their proportion in a sample is less than $0.1 \%$. To achieve this sensitivity, some optimization steps (primer/probe design, thermal profiles) may be necessary. Sequencing methods can also be used, but require specific considerations, in particular concerning adequate coverage. Similarly, the necessary equipment for ddPCR is increasingly available in these laboratories. In addition, ddPCR is already being used for similar applications, like qPCR. For ddPCR, the requirements for the technical equipment are higher than those for qPCR.

Most PCR methods can be carried out with existing technical equipment and by existing staff of a GMO enforcement and official control laboratory; however, for the evaluation of the data, employees with appropriate expertise are required. Due to specific regulatory requirements [7], even more resources are needed when using sequencing in enforcement control. Although the necessary "wet lab" activities can be carried out by existing staff, the generation and processing of the sequencing data creates new data sets with additional storage and archiving requirements [36]. Additional staff with the appropriate expertise in bioinformatic analysis are required for the evaluation of the data. For the provision and maintenance of the necessary IT infrastructure, personnel with knowledge of relevant software solutions and their implementation in the laboratoryspecific IT environment is essential.

In order to ensure that the detection methods used provide correct results, such measurement methods need to be subject to appropriate validation. Guidelines for the validation of qPCR methods in GMO analysis are available [22,37], whereas no guidelines for validation of methods for the detection of genome-edited plants and products have been published. As validation solely deals with the technical performance criteria of a method, the existing guidelines can also be applied to the validation of qPCR methods used for detection of genome-edited material. Concerning ddPCR, general requirements and guidelines for the application of ddPCR are under development by various technical committees of the International Organization for Standardization (ISO) (including ISO 20395:2019). In addition, JRC has published a guidance document on the use of ddPCR in GMO analysis [38]. Based on these guidelines, validation measures for the application of ddPCR for genome-edited plants and products can be defined. To enable enforcement control according to the regulations and standards, it is necessary to establish standardized requirements for individual laboratory validation. Based on this validation, the methods can be accredited according to ISO 17025 [21]. 
The availability of reference material is a crucial point when attempting to detect and quantify genome-edited material. In the case of an EU market authorization, the applicant is obliged to provide reference materials and a method to detect the product under examination [3]. However, in the case that no application for approval in the EU has been submitted and the product is marketed elsewhere outside the EU, the availability of appropriate reference material must be arranged with the developer, and an adequate detection method should be made accessible. A system to ensure the availability of reference material and methods must be established, regardless of whether the genome-edited plant or product is authorized or not.

It is usually not part of the tasks of an enforcement laboratory to develop methods. However, the participation of experts from enforcement laboratories in projects, possibly under the aegis of the JRC, is desirable if the necessary resources are made available. The prerequisite to develop methods to detect genome-edited plants is adequate information, which is provided by developers in the course of the authorization process. Alternatively, databases such as EUginius [39] are a source of relevant information, provided that they also take into account data from countries where genome-edited plants are not regulated, and on the condition that they are kept up to date. The competent authorities of the EU member states could promote the global exchange of information between regulatory institutions, thereby supporting their enforcement laboratories.

\section{Conclusions}

To date, the detection of GMOs is typically based on qPCR, a method widely used in official enforcement laboratories to target DNA. The analyses of existing and upcoming methods showed that SNVs are reliably detected, providing the technical possibility of tracking genome-edited plants. These PCR methods can usually be carried out with the available technical equipment and by existing staff in a GMO control laboratory. However, an analytical method for the detection of a genome-edited product should be found fit for the intended purpose by the EURL before it is applied for official control purposes by enforcement laboratories.

The prerequisite for integrating the new methods into the current testing process is the availability of established detection methods and adequate reference material. In order to allow easy access for enforcement laboratories to all relevant information, it is recommended to establish an international NGT method database. Furthermore, in order to enable method development and to examine the characteristics of applications by genome editing, action is needed to finance research activities on both the international and European levels. In addition, guidelines for the validation of detection methods must be developed to fulfill the regulatory requirements and to ensure transferability of the methods across laboratories. Such activity should include sequencing as promising alternative methodology in the near future.

Author Contributions: Conceptualization, A.R., F.N. and R.H.; Investigation, F.N., R.H. and W.S.; Writing-original draft preparation, A.R., F.N., R.H.; Writing-review and editing, A.R., R.H., F.N. and W.S.; Project management, A.R. All authors have read and agreed to the published version of the manuscript.

Funding: This project was commissioned by the German Federal Agency for Nature Conservation (BfN) Research \& Development Grant No. (FKZ) 3519801000 ("Nachweismethoden für genomeditierte und klassische GV-Pflanzen"). It was funded by the German Federal Ministry for the Environment, Nature Conservation and Nuclear Safety (BMU).

Institutional Review Board Statement: Not applicable.

Informed Consent Statement: Not applicable.

Data Availability Statement: No new data were created or analyzed in this study. Data sharing is not applicable to this article.

Conflicts of Interest: The authors declare no conflict of interest. 


\section{References}

1. European Commission. GMO Legislation. Available online: https://ec.europa.eu/food/plant/gmo/legislation_en (accessed on 25 November 2020).

2. European Commission. Directive 2001/18/EC of the European Parliament and of the Council of 12 March 2001 on the deliberate release into the environment of genetically modified organisms and repealing Council Directive 90/220/EEC. Off. J. Eur. Communities 2001, 106, 1-38.

3. European Commission. Regulation (EC) No. 1829/2003 of the European Parliament and of the Council of 22 September 2003 on genetically modified food and feed. Off. J. Eur. Union 2003, L268, 1-23.

4. European Commission. Regulation (EC) No. 1830/2003 of the European Parliament and of the Council of 22 September 2003 concerning the traceability and labelling of genetically modified organisms and the traceability of food and feed products produced from genetically modified organisms and amending Directive 2001/18/EC. Off. J. Eur. Union 2003, L268, $24-28$.

5. European Commission. Directive 2009/41/EC of the European Parliament and of the Council of 6 May 2009 on the contained use of genetically modified micro-organisms. Off. J. Eur. Union 2009, L125, 75-97.

6. European Commission. Commission Regulation (EU) No. 619/2011 of 24 June 2011 laying down the methods of sampling and analysis for the official control of feed as regards presence of genetically modified material for which an authorisation procedure is pending or the authorisation of which has expired. Off. J. Eur. Union 2011, L166, 9-15.

7. European Commission. Regulation (EU) 2017/625 of the European Parliament and of the Council of 15 March 2017 on official controls and other official activities performed to ensure the application of food and feed law, rules on animal health and welfare, plant health and plant protection products, amending Regulations (EC) No. 999/2001, (EC) No. 396/2005, (EC) No. 1069/2009, (EC) No. 1107/2009, (EU) No. 1151/2012, (EU) No. 652/2014, (EU) 2016/429 and (EU) 2016/2031 of the European Parliament and of the Council, Council Regulations (EC) No. 1/2005 and (EC) No. 1099/2009 and Council Directives 98/58/EC, 1999/74/EC, 2007/43/EC, 2008/119/EC and 2008/120/EC, and repealing Regulations (EC) No. 854/2004 and (EC) No. 882/2004 of the European Parliament and of the Council, Council Directives 89/608/EEC, 89/662/EEC, 90/425/EEC, 91/496/EEC, 96/23/EC, 96/93/EC and 97/78/ EC and Council Decision 92/438/EEC (Official Controls Regulation). Off. J. Eur. Union 2017, L95, 1-142.

8. Broothaerts, W.; Cordeiro, F.; Robouch, P.; Emons, H. Ten years of proficiency testing reveals an improvement in the analytical performance of EU National Reference Laboratories for genetically modified food and feed. Food Control 2020, $114,107237$. [CrossRef]

9. Court of Justice of the European Union. InfoCuria. Case-law ECLI:EU:C:2018:583. Judgement of the Court (Grand Chamber) 25 July 2018. Available online: http:/ / curia.europa.eu/juris/document/document.jsf?docid=204387\&text=\&dir=\&doclang=EN\& part $=1 \&$ occ $=$ first \&mode $=$ DOC\&pageIndex $=0 \& c i d=2442882$ (accessed on 25 November 2020).

10. European Commission. EC Study on New Genomic Techniques. Available online: https://ec.europa.eu/food/plant/gmo/ modern_biotech/new-genomic-techniques_en (accessed on 25 November 2020).

11. Razzaq, A.; Saleem, F.; Kanwal, M.; Mustafa, G.; Yousaf, S.; Imran Arshad, H.M.; Hameed, M.K.; Khan, M.S.; Joyia, F.A. Modern trends in plant genome editing: An inclusive review of the CRISPR/Cas9 toolbox. Int. J. Mol. Sci. 2019, 20, 4045. [CrossRef]

12. Holst-Jensen, A.; Bertheau, Y.; de Loose, M.; Grohmann, L.; Hamels, S.; Hougs, L.; Morisset, D.; Pecoraro, S.; Pla, M.; Van den Bulcke, M.; et al. Detecting un-authorized genetically modified organisms (GMOs) and derived materials. Biotechnol. Adv. 2012, 30, 1318-1335. [CrossRef]

13. Petrillo, M.; Angers-Loustau, A.; Henriksson, P.; Bonfini, L.; Patak, A.; Kreysa, J. JRC GMO-Amplicons: A collection of nucleic acid sequences related to genetically modified organisms. Database 2015, 2015, bav101. [CrossRef]

14. Angers-Loustau, A.; Petrillo, M.; Bonfini, L.; Gatto, F.; Rosa, S.; Patak, A.; Kreysa, J. JRC GMO-Matrix: A web application to support Genetically Modified Organisms detection strategies. BMC Bioinform. 2014, 15, 1-9. [CrossRef]

15. Bonfini, L.; Van den Bulcke, M.H.; Mazzara, M.; Ben, E.; Patak, A. GMOMETHODS: The European Union database of reference methods for GMO analysis. J. AOAC Int. 2012, 95, 1713-1719. [CrossRef] [PubMed]

16. Broeders, S.R.; De Keersmaecker, S.C.; Roosens, N.H. How to deal with the upcoming challenges in GMO detection in food and feed. J. Biomed. Biotechnol. 2012, 2012, 402418. [CrossRef]

17. Verginelli, D.; Paterno, A.; De Marchis, M.L.; Quarchioni, C.; Vinciguerra, D.; Bonini, P.; Peddis, S.; Fusco, C.; Misto, M.; Marfoglia, C.; et al. Development and comparative study of a pat/bar real-time PCR assay for integrating the screening strategy of a GMO testing laboratory. J. Sci. Food Agric. 2020, 100, 2121-2129. [CrossRef] [PubMed]

18. Basso, M.F.; Arraes, F.B.M.; Grossi-de-Sa, M.; Moreira, V.J.V.; Alves-Ferreira, M.; Grossi-de-Sa, M.F. Insights into genetic and molecular elements for transgenic crop development. Front. Plant Sci. 2020, 11, 509. [CrossRef] [PubMed]

19. Grohmann, L.; Keilwagen, J.; Duensing, N.; Dagand, E.; Hartung, F.; Wilhelm, R.; Bendiek, J.; Sprink, T. Detection and identification of genome editing in plants: Challenges and opportunities. Front. Plant Sci. 2019, 10, 236. [CrossRef] [PubMed]

20. Chhalliyil, P.; Ilves, H.; Kazakov, S.A.; Howard, S.J.; Johnston, B.H.; Fagan, J. A real-time quantitative PCR method specific for detection and quantification of the first commercialized genome-edited plant. Foods 2020, 9, 1245. [CrossRef]

21. International Organization for Standardization. ISO/IEC 17025:2017. General Requirements for the Competence of Testing and Calibration Laboratories. Available online: https:/ /www.iso.org/standard/66912.html (accessed on 25 November 2020).

22. European Network of GMO Laboratories. ENGL-Definition of Minimum Performance Requirements for Analytical Methods of GMO Testing. Available online: https:/ / gmo-crl.jrc.ec.europa.eu/doc/MPR\%20Report\%20Application\%2020_10_2015.pdf (accessed on 25 November 2020). 
23. Van den Eede, G.; Kay, S.; Anklam, E.; Schimmel, H. Analytical challenges: Bridging the gap from regulation to enforcement. J. AOAC Int. 2002, 85, 757-761. [CrossRef]

24. Johnson, M.P.; Haupt, L.M.; Griffiths, L.R. Locked nucleic acid (LNA) single nucleotide polymorphism (SNP) genotype analysis and validation using real-time PCR. Nucleic Acids Res. 2004, 32, e55. [CrossRef]

25. Mouritzen, P.; Nielsen, A.T.; Pfundheller, H.M.; Choleva, Y.; Kongsbak, L.; Møller, S. Single nucleotide polymorphism genotyping using locked nucleic acid (LNA ${ }^{\mathrm{TM}}$ ). Expert Rev. Mol. Diagn. 2003, 3, 27-38. [CrossRef]

26. Ugozzoli, L.A.; Latorra, D.; Pucket, R.; Arar, K.; Hamby, K. Real-time genotyping with oligonucleotide probes containing locked nucleic acids. Anal. Biochem. 2004, 324, 143-152. [CrossRef]

27. You, Y.; Moreira, B.; Behlke, M.; Owczarzy, R. Design of LNA probes that improve mismatch discrimination. Nucleic Acids Res. 2006, 34, e60. [CrossRef] [PubMed]

28. Dobosy, J.R.; Rose, S.D.; Beltz, K.R.; Rupp, S.M.; Powers, K.M.; Behlke, M.A.; Walder, J.A. RNase H-dependent PCR (rhPCR): Improved specificity and single nucleotide polymorphism detection using blocked cleavable primers. BMC Biotechnol. 2011, 11, 80. [CrossRef] [PubMed]

29. Ayalew, H.; Tsang, P.W.; Chu, C.; Wang, J.; Liu, S.; Chen, C.; Ma, X.-F. Comparison of TaqMan, KASP and rhAmp SNP genotyping platforms in hexaploid wheat. PLoS ONE 2019, 14, e0217222. [CrossRef] [PubMed]

30. Broccanello, C.; Chiodi, C.; Funk, A.; McGrath, J.M.; Panella, L.; Stevanato, P. Comparison of three PCR-based assays for SNP genotyping in plants. Plant Methods 2018, 14, 28. [CrossRef] [PubMed]

31. Findlay, S.D.; Vincent, K.M.; Berman, J.R.; Postovit, L.-M. A digital PCR-based method for efficient and highly specific screening of genome edited cells. PLoS ONE 2016, 11, e0153901. [CrossRef] [PubMed]

32. Miyaoka, Y.; Chan, A.H.; Judge, L.M.; Yoo, J.; Huang, M.; Nguyen, T.D.; Lizarraga, P.P.; So, P.-L.; Conklin, B.R. Isolation of single-base genome-edited human iPS cells without antibiotic selection. Nat. Methods 2014, 11, 291-293. [CrossRef]

33. Mock, U.; Hauber, I.; Fehse, B. Digital PCR to assess gene-editing frequencies (GEF-dPCR) mediated by designer nucleases. Nat. Protoc. 2016, 11, 598-615. [CrossRef]

34. Bruge, F.; Littarru, G.P.; Silvestrini, L.; Mancuso, T.; Tiano, L. A novel Real Time PCR strategy to detect SOD3 SNP using LNA probes. Mutat. Res. 2009, 669, 80-84. [CrossRef]

35. Wenger, A.M.; Peluso, P.; Rowell, W.J.; Chang, P.C.; Hall, R.J.; Concepcion, G.T.; Ebler, J.; Fungtammasan, A.; Kolesnikov, A.; Olson, N.D.; et al. Accurate circular consensus long-read sequencing improves variant detection and assembly of a human genome. Nat. Biotechnol. 2019, 37, 1155-1162. [CrossRef]

36. Wang, X. Next-Generation Sequencing Data Analysis; CRC Press, Taylor \& Francis Group: Boca Raton, FL, USA, 2016 ; p. 258.

37. Grohmann, L.; Broll, H.; Dagand, E.; Hildebrandt, S.; Hübert, P.; Kiesecker, H.; Lieske, K.; Mäde, D.; Mankertz, D.; Reiting, D.; et al. Guidelines for the Single-Laboratory Validation of Qualitative Real-Time PCR Methods; Bundesamt für Verbraucherschutz und Lebensmittelsicherheit (BVL): Braunschweig, Germany, 2016; p. 17. Available online: https: / www.bvl.bund.de/SharedDocs/ Downloads/07_Untersuchungen/Guidelines\%20for\%20the\%20single\%20laboratory.pdf (accessed on 25 November 2020).

38. Joint Research Centre. JRC-Overview and Recommendations for the Application of Digital PCR. Available online: https:/ / www.researchgate.net/profile/Philippe_Corbisier/publication/333079273_Overview_and_recommendations_ for_the_application_of_digital_PCR_European_Network_of_GMO_Laboratories_ENGL/links/5cda663ca6fdccc9ddaab3f4 /Overview-and-recommendations-for-the-application-of-digital-PCR-European-Network-of-GMO-Laboratories-ENGL.pdf (accessed on 25 November 2020).

39. EUginius. European GMO Initiative for a Unified Database System. Detection Methods. Available online: https://www.euginius. eu/euginius/pages/detection_index.jsf;jsessionid=Pa2i0zseD2UMYV1ZQu75Db6nqEwOYiJnCt9MZAsu.subs262 (accessed on 25 November 2020). 